\title{
TRANSVERSITY SIGNALS IN TWO HADRON CORRELATION AT COMPASS
}

\author{
RAINER JOOSTEN \\ ON BEHALF OF THE COMPASS COLLABORATION \\ Helmholtz Institut für Strahlen- und Kernphysik \\ Nußallee 14-16, 53115 Bonn, Germany \\ E-mail: Rainer.Joosten@cern.ch
}

\begin{abstract}
Measurement of two hadron production introducing the chiral odd interference fragmentation function $H_{1}^{\triangleleft}$ is considered a new probe of the transverse spin distribution $\Delta_{T} q(x)$. COMPASS is a fixed target experiment on the SPS M2 beamline at CERN. Its target can be polarised both longitudinally and transversally with respect to the polarised $160 \mathrm{GeV} / \mathrm{c} \mu^{+}$beam. In 2002, 2003, and $2004,20 \%$ of the beam-time was spent in the transverse configuration on a ${ }^{6} \mathrm{LiD}$ target, allowing the measurement of transversity effects. First results of the analysis of two hadron production will be reported.
\end{abstract}

\section{Theoretical background}

The cross-section for deep inelastic scattering off spin $1 / 2$ hadrons can be parametrised, in leading order, in terms of three quark distribution functions: the helicity averaged distribution $q(x)$, the longitudinal helicity distribution $\Delta q(x)$, and the transverse spin distribution $\Delta_{T} q(x)$. This last distribution function, referred to as transversity, is chiral-odd and can only be measured in combination with another chiral-odd function. So far, attempts were made to measure $\Delta_{T} q(x)$ in combination with the Collins fragmentation-function $\Delta D_{a}^{h}\left(z, p_{T}^{h}\right)$, requiring the partial detection of the hadronic products (semi-inclusive measurement) [1]. Another suggested and very promising probe is the measurement of two hadron production introducing the chiral odd interference fragmentation function $H_{1}^{\varangle}\left(z, M_{h}^{2}\right)$. The properties of interference fragmentation functions are described in Refs. $[2,3,4,5,6]$.

At leading twist, the fragmentation function of a quark q into a pair $\mathrm{h}$ of two hadrons $h_{1}$ and $h_{2}$ can be written as:

$$
D_{q}^{h}\left(z, M_{h}^{2}\right)+H_{1}^{\varangle}\left(z, M_{h}^{2}\right) \sin \left(\varphi_{R S}\right)
$$




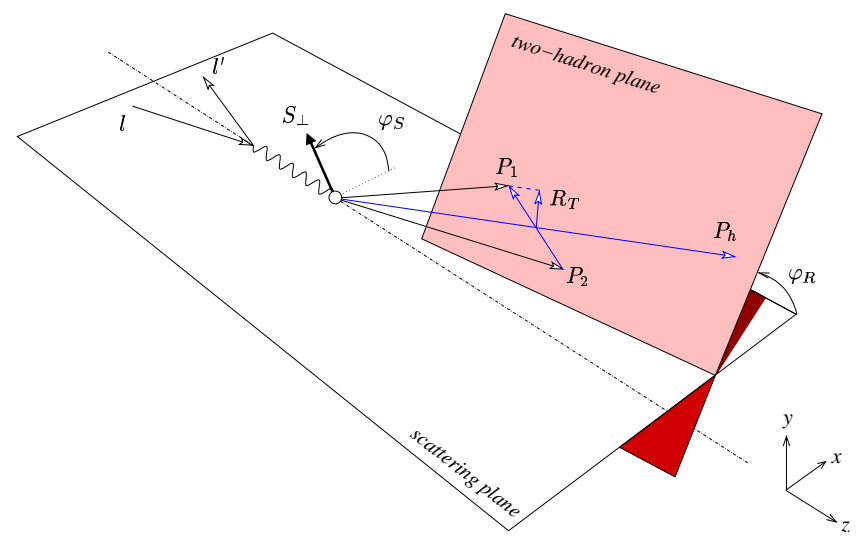

Figure 1. Description of the angles involved in the measurement of single spin asymmetries in deep-inelastic production of two hadrons (from Ref. [7])

with $\varphi_{R S}=\varphi_{R}-\varphi_{S^{\prime}}=\varphi_{R}+\varphi_{S}-\pi$, where $\varphi_{S^{\prime}}$ is the azimuthal angle of the struck quark, $\varphi_{S}$ is the azimuthal angle of the initial quark spin and $\varphi_{R}$ the angle of the hadron plane in the lepton scattering plane and $\varphi_{S^{\prime}}=\pi-\varphi_{S}$. $z=z_{1}+z_{2}=\left(E\left(h_{1}\right)+E\left(h_{2}\right)\right) /\left(E_{l}-E_{l^{\prime}}\right), E_{l}$ being the incoming and $E_{l^{\prime}}$ the scattered lepton energy, is the fraction of the transfered energy carried by the two hadrons, and $M_{h}^{2}$ is their invariant mass squared. The angles are defined according to Ref. [7] (see Fig. 1), which follows the so-called Trento conventions.

Therefore, an asymmetry is expected in the azimuthal angle of the hadron plane which depends on $\varphi_{R S}$. This asymmetry, which gives information on the transversity distribution, has not been measured so far on a transversely polarised target. Even a strong dependance of the fragmentation function $H_{1}^{\varangle}\left(z, M_{h}^{2}\right)$ on the invariant mass of the two-hadron system has been predicted in the region of the $\rho$-mass (Ref. [4]) due to an interference term in two pion production.

The measured raw asymmetry $A_{U T}^{s i n \varphi_{R S}}$ is connected to the physically relevant asymmetry $A_{\varphi_{R S}}$ by

$$
\frac{A_{U T}^{s i n \varphi_{R S}}}{D_{N N} f P}=A_{\varphi_{R S}}=\frac{\Sigma_{i} e_{i} \Delta_{T} q_{i}(x) H_{1}^{\varangle h}\left(z, M_{h}^{2}\right)}{\Sigma_{i} e_{i} q_{i}(x) D_{i}^{h}\left(z, M_{h}^{2}\right)}
$$

where $f(\approx 0.4)$ is the dilution factor, $P(\approx 0.45)$ the target polarisation and $D_{N N}=(1-y) /\left(1-y+y^{2} / 2\right)$ the depolarisation factor. Here, $y=$ $\left(E_{l}-E_{l^{\prime}}\right) / E_{l}$ is the fraction of the incoming lepton energy transfered to the hadronic system. 


\section{Results from the COMPASS 2002 transversity run}

The COMPASS experiment [8,9] uses the high intensity $160 \mathrm{GeV}$ secondary $\mu^{+}$-beam from $\pi$-decay in the CERN SPS M2 beamline. This beam is naturally longitudinally polarised with a polarisation of $\approx-76 \%$.

The polarised target consists of two subsequent target cells filled with ${ }^{6} \mathrm{LiD}$, each $60 \mathrm{~cm}$ long, which can be individually polarised using separate RF-cavities. This allows to take data simultaneously on two target cells of opposite polarisation. The target can be polarised longitudinally or transversely with respect to the beam axis.

The data discussed here was taken in 2002 with a transversely polarised target. The sample consists of two data taking periods of approximately five days, where each period was split in two subperiods with opposite spin orientation in the individual target cells.

The event selection is analogous to the analysis of the Collins and Sivers asymmetries [1]. The primary vertex, with identified incoming and scattered muon, is required to be in either of the two target cells. At least two hadrons are required to origin from the same vertex. The separation of muons and hadrons is primarily done by cutting on the amount of traversed material in the spectrometer. Moreover, the kinematic cuts $Q^{2}>1(\mathrm{GeV} / \mathrm{c})^{2}, W>5 \mathrm{GeV} / \mathrm{c}^{2}$ and $0.1<y<0.9$ were applied to ensure a deep-inelastic scattering sample above nuclear resonances and within the COMPASS trigger acceptance. The final data sample had average values for $x=0.034, y=0.33$, and $Q^{2}=2.7(G e V / c)^{2}$. The mean hadron multiplicity of the events selected by these kinematic cuts is 1.9 hadrons/event. As a first step, we selected the leading and next to leading hadron for further analysis. Here, the leading hadron is determined as the most energetic non-muonic charged particle of the primary vertex having $z>0.25$. For the next to leading hadron, being the second most energetic non-muonic charged particle of the primary vertex, no $z$ cut is applied.

From the data, for each target cell and polarisation, the property

$$
N\left(\varphi_{R S}\right)=N_{0} \cdot\left(1+A_{U T}^{\sin \varphi_{R S}} \cdot \sin \varphi_{R S}\right) \cdot F_{a c c}\left(\varphi_{R S}\right)
$$

can be derived, where $F_{a c c}\left(\varphi_{R S}\right)$ is the (unknown) angle dependant acceptance function of the detector. However, by comparing the subperiods with opposite target spin, this acceptance function cancels, resulting in

$$
A_{U T}^{\sin \varphi_{R S}} \cdot \sin \varphi_{R S}=\frac{N^{\Uparrow}\left(\varphi_{R S}\right)-R N^{\Downarrow}\left(\varphi_{R S}\right)}{N^{\Uparrow}\left(\varphi_{R S}\right)+R N^{\Downarrow}\left(\varphi_{R S}\right)}
$$

where $R=N_{\text {tot }}^{\Uparrow} / N_{\text {tot }}^{\Downarrow}$ is the ratio of the events with opposite target polar- 


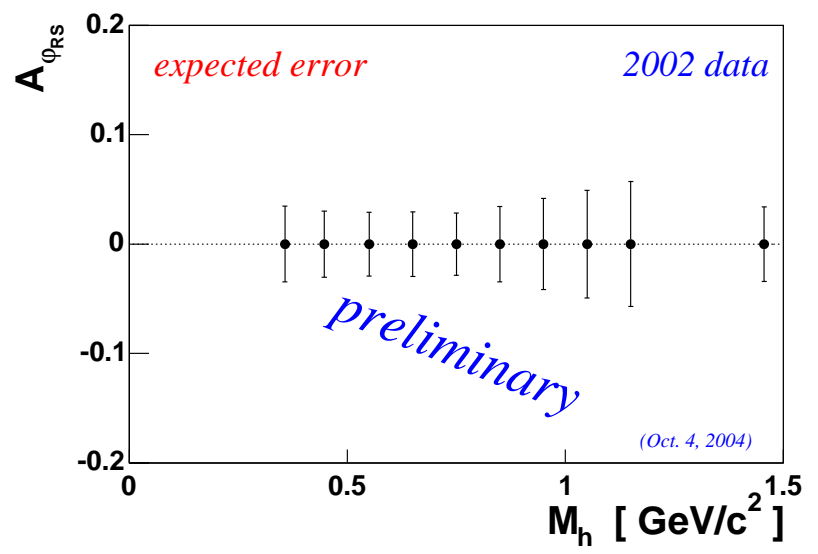

Figure 2. Estimated error of the asymmetries $A_{\varphi_{R S}}$ for the 2002 data vs. invariant mass of the hadron pair. A binning of $100 \mathrm{MeV}$ has been chosen except for the last point which covers the range from $1.2 \mathrm{GeV}$ to $2.0 \mathrm{GeV}$

isation. This procedure is repeated for both target cells and both periods. Finally, the weighted mean of the results is calculated.

The present status of the analysis does not yet allow to give results on the asymmetries. However, based on the first analysis and statistics an error estimate on the 2002 data can be given. Fig. 2 shows the expected errors $\Delta A_{\varphi_{R S}}=\Delta A_{U T}^{\sin \varphi_{R S}} / D_{N N} f P$ vs. the invariant mass $M_{h}$ of the hadron pair.

\section{References}

1. P. Pagano (COMPASS), these proceedings.

2. J.R. Collins, S.F. Heppelmann and G.A. Ladinsky,Nucl. Phys. B420, 565 (1994).

3. X. Artru and J. C. Collins, Z. Phys. C69, 277 (1996).

4. R. L. Jaffe, X. Jin and J. Tang, Phys. Rev. Lett. 80, 1166 (1998).

5. A. Bianconi, S. Boffi, R. Jakob and M. Radici, Phys. Rev. D62, 034008 (2000).

6. A. Bacchetta and M. Radici, Phys. Rev. D69, 074026 (2004).

7. A. Bacchetta and M. Radici, Proceeding of DIS 2004, hep-ph/0407345 (2004).

8. G. Baum et al. [COMPASS Collaboration], CERN-SPSLC-96-14.

9. A. Bressan, "Recent results from COMPASS", these proceedings. 